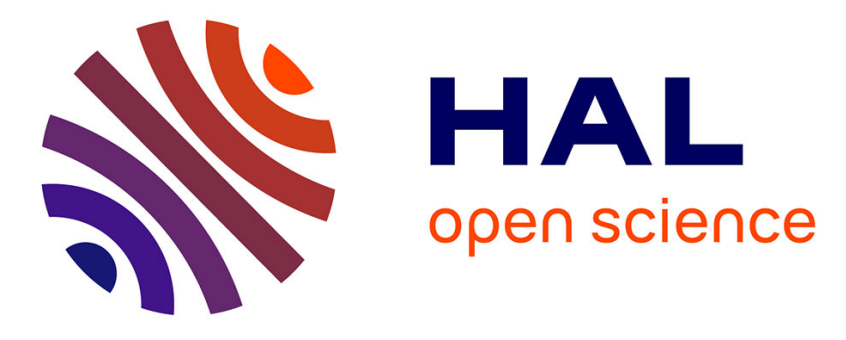

\title{
Spectral Adaptation Transform for Multispectral Constancy
}

Haris Ahmad Khan, Jean-Baptiste Thomas, Jon Yngve Hardeberg, Olivier Laligant

\section{- To cite this version:}

Haris Ahmad Khan, Jean-Baptiste Thomas, Jon Yngve Hardeberg, Olivier Laligant. Spectral Adaptation Transform for Multispectral Constancy. Journal of Imaging Science and Technology, 2018, 62 (2), pp.205041 - 2050412. 10.2352/J.ImagingSci.Technol.2018.62.2.020504 . hal-01781434

\section{HAL Id: hal-01781434 https://hal.science/hal-01781434}

Submitted on 30 Apr 2018

HAL is a multi-disciplinary open access archive for the deposit and dissemination of scientific research documents, whether they are published or not. The documents may come from teaching and research institutions in France or abroad, or from public or private research centers.
L'archive ouverte pluridisciplinaire HAL, est destinée au dépôt et à la diffusion de documents scientifiques de niveau recherche, publiés ou non, émanant des établissements d'enseignement et de recherche français ou étrangers, des laboratoires publics ou privés. 


\title{
Spectral adaptation transform for multispectral constancy
}

\author{
Haris Ahmad Khan ${ }^{\mathrm{a}, \mathrm{b}, *}$, Jean-Baptiste Thomas ${ }^{\mathrm{a}, \mathrm{b}}$, Jon Yngve Hardeberg ${ }^{\mathrm{a}}$, \\ Olivier Laligant ${ }^{\mathrm{b}}$ \\ ${ }^{a}$ The Norwegian Colour and Visual Computing Laboratory. \\ NTNU - Norwegian University of Science and Technology, Gjøvik, Norway \\ ${ }^{b}$ Le2i, FRE CNRS 2005, Université Bourgogne Franche-Comté, Dijon, France
}

\begin{abstract}
The spectral reflectance of an object surface provides valuable information of its characteristics. Reflectance reconstruction from multispectral image data is typically based on certain assumptions. One of these common assumptions is that the same illumination is used for system calibration and image acquisition. We propose the concept of multispectral constancy which transforms the captured sensor data into an illuminant-independent representation, analogously to the concept of computational color constancy. We propose to transform the multispectral image data to a canonical representation through spectral adaptation transform. The performance of such a transform is tested on measured reflectance spectra and hyperspectral reflectance images. We also investigate the robustness of the transform to the inaccuracy of illuminant estimation in natural scenes. Results of reflectance reconstruction show that the proposed spectral adaptation transform is efficient and is robust to error in illuminant estimation.
\end{abstract}

Keywords: Multispectral imaging, reflectance reconstruction, multispectral constancy, illuminant estimation, spectral adaptation transform.

\footnotetext{
${ }^{*}$ Corresponding author

Email address: Haris-Ahmad.Khan@u-bourgogne.fr (Haris Ahmad Khan)
} 


\section{Introduction}

The formation of an image depends on the spectral reflectance of the surface being viewed, the spectral power distribution and intensity of the illumination, and the spectral sensitivities of the sensors. In the human visual system, the three cone types act as sensors. In case of a camera with 3 channels, the RGB filters together with the imaging sensor, play a similar role. The human visual system has the natural ability to perceive constant color of surfaces despite the change in spectral composition of the illuminant, and this ability to discard illumination effects is called Color Constancy [1]. Creation of such models for illuminant invariant representation of scenes in computer vision is called computational color constancy [2]. An illuminant invariant representation is important for computer vision applications including object recognition, tracking and image classification [3]. There are two major techniques for achieving computational color constancy. One method is to compute illuminant invariant features, and a second method is to estimate the illuminant and later apply a correction [4]. In this paper, we use the former method of illuminant estimation.

The advancement in sensor technology has developed the use of multispectral imaging for indoor and close range imaging. The ability of multispectral imaging in acquisition of more spectral information is useful for object and material classification and identification by means of spectral reconstruction $[5,6,7,8,9]$ of surfaces in a scene. The need for spectral reconstruction of surfaces was recognized in 1980s $[10,11,12,13,14]$. Since then, many methods are developed to provide spectral reconstruction from the camera data. Most of these methods rely on the use of training data to learn the mapping between the camera data and the desired spectra. This process is called calibration of the system and is performed through a training set of measured reflectances and radiance data with a given illuminant. To maintain a reasonable accuracy, the same illuminant 
is required for scene acquisition. This limitation of having the same illuminant for calibration and image acquisition is a major shortcoming for generic use of multispectral imaging [15].

To overcome this limitation, we propose to transform the acquired multispectral data under any unknown illumination into its canonical representation. This transformation requires the estimation of scene illuminant. For multispectral images, estimation of illuminant in the sensor domain by using image statistics based methods is proposed by Thomas [16] and Khan et al. [17]. In their work, the illuminant estimation methods for color images are extended from three channels to $K$ channels. The estimated illuminant is used for the diagonal correction [18] to transform the input sensor data into a canonical representation. We call this consistent representation of multispectral data as multispectral constancy. A preliminary proposal of this concept is provided in [19].

To achieve multispectral constancy, we use a diagonal transform and also introduce a spectral adaptation transform (SAT). The concept of SAT being proposed is closely related to the data based sensor sharpening transform by Finlayson et al. [20]. The main difference between these strategies is that instead of finding a sharpening transform and then diagonalizing it, we want to optimize the result after applying the diagonal transform, where the elements of diagonal transform are obtained from illuminant estimation in the sensor domain. The proposed idea of multispectral constancy and optimization of diagonal transform is tested with a simulated multispectral camera on the reflectance dataset and hyperspectral images of real scenes from the Foster dataset [21]. The advantage of having a canonical representation is that the spectral reconstruction system can be calibrated with a canonical illuminant and hence, the condition of having same illuminants for training under which the scene is 
acquired, is no longer needed. Results show a promising aspect of the use of multispectral imaging for outdoor scene acquisition under uncontrolled illumination condition.

This paper is organized as follows. In Section 2, we define multispectral constancy after formalizing a model for the multispectral image acquisition system. System calibration and spectral reconstruction are defined as linear problem in the section. Section 3 defines our experimental protocol based on simulations. Results are analyzed in Section 4 before we conclude.

\section{Multispectral constancy}

\subsection{Definition}

In a simplified noiseless imaging model, formation of an image depends on the spectral sensitivity of imaging sensor $c(\lambda)$, spectral reflectance of the surface $r(\lambda)$ and the spectral power distribution of illuminant $e(\lambda)$. This formation for the visible wavelength spectrum $\omega$ is defined as

$$
f=\int_{\omega} r(\lambda) e(\lambda) c(\lambda) d \lambda
$$

In practice, we can formulate an extended and discrete version of Eq. 1 as;

$$
\mathbf{F}=\mathbf{R E C}
$$

Considering the spectral sampling $(N)$ of $10 \mathrm{~nm}$ within the wavelength range of $400 \mathrm{~nm}$ to $700 \mathrm{~nm}$ and $K$ number of spectral channels, $\mathbf{F}$ is $S \times K$ matrix ( $S$ is the number of spectral samples), $\mathbf{R}$ is $S \times N$ matrix of surface reflectance, $\mathbf{E}$ is the diagonal matrix $(N \times N)$ of the scene illuminant and $\mathbf{C}$ is $N \times K$ matrix, consisting of spectral sensitivities of the channels. 
Here we consider two cases of image acquisition with the same imaging system. One image is acquired with a canonical illumination $\mathbf{E}_{c}$ and another image with an unknown illuminant $\mathbf{E}_{\text {ill }}$. We present both cases in parallel in Eq. 3.

$$
\mathbf{F}_{i l l}=\mathbf{R E}_{i l l} \mathbf{C} \quad ; \quad \mathbf{F}_{c}=\mathbf{R E}_{c} \mathbf{C}
$$

To perform the spectral reflectance estimation $\hat{\mathbf{R}}$ from the imaged data in both of the above mentioned cases, a generalized inverse, denoted by ${ }^{+}$, can be applied as;

$$
\hat{\mathbf{R}}=\mathbf{F}_{i l l} \mathbf{C}^{+} \mathbf{E}_{i l l}^{+} \quad ; \quad \hat{\mathbf{R}}=\mathbf{F}_{c} \mathbf{C}^{+} \mathbf{E}_{c}^{+}
$$

However, $\mathbf{C}$ is not necessarily a square matrix and therefore, it is not trivial to compute the inverse. Computational procedures are applied to achieve the task of spectral reconstruction. There are several works in literature where the spectral reconstruction is performed by linear transform through a calibration matrix. Linear methods are popular for learning the mapping between camera data and desired output (spectral reflectance). The idea behind using linear methods is that when the reflectance spectra is continuous and band-limited [10, 13], the statistical analysis of measured reflectances of standard color samples are enough to calibrate the spectral reconstruction system $[14,22]$.

By using the calibration matrix $\mathbf{W}$, the equations for spectral reflectance reconstruction become $\hat{\mathbf{R}}=\mathbf{F}_{i l l} \mathbf{W}_{\text {ill }}$ and $\hat{\mathbf{R}}=\mathbf{F}_{c} \mathbf{W}_{c}$ for both cases, respectively. This calibration is specific for a given illumination $\mathbf{E}_{c}$. The scene illuminant plays a direct role in the spectral reconstruction [7, 23], because of its effect in the camera image formation, as in Eq. 1.

With $\mathbf{F}_{c}$, the spectral reconstruction system can work efficiently, since the calibration is already performed with the same canonical illuminant, but with $\mathbf{F}_{i l l}$, the calibration matrix needs to be recomputed with $\mathbf{E}_{i l l}$. Measurement 
of scene illuminant and calibration matrix for each change in imaging environment is a difficult task and is not a practical solution for a situation where the illumination is not constant all the time. To avoid this problem, we propose to transform the acquired multispectral data $\mathbf{F}_{i l l}$ into a canonical representation $\mathbf{F}_{c}$. Thus, $\mathbf{W}_{c}$ can be used for the spectral reconstruction from the multispectral data, being taken under any illumination. We denote such a transform as $\mathbf{M}^{c, i l l}$, which maps the camera data $\mathbf{F}_{i l l}$, taken under unknown illuminant $\mathbf{E}_{i l l}$, into its canonical representation $\mathbf{F}_{c}$.

$$
\mathbf{F}_{c}=\mathbf{M}^{c, i l l} \mathbf{F}_{i l l}
$$

Once such a transform is available, then the problem of spectral reconstruction is limited to finding the transform $\mathbf{M}^{c, i l l}$. The spectral reconstruction in this case is mathematically represented as;

$$
\hat{\mathbf{R}}=\mathbf{W}_{c} \mathbf{M}^{c, i l l} \mathbf{F}_{i l l}
$$

We call this concept of illuminant invariant representation of multispectral data as multispectral constancy. By achieving multispectral constancy, the requirement of having the same illumination for calibration and scene is no longer required.

In this work, we propose the use of illuminant estimation for achieving multispectral constancy. In [19], a diagonal transform is used as $\mathbf{M}^{c, \text { ill }}$ and the preliminary results for spectral reconstruction from the sensor data were provided. Such a transform requires the estimation of scene illuminant in the sensor domain and the sensor data is corrected from effects of illumination through a diagonal transform $(\mathbf{D})$. The diagonal matrix $\mathbf{D}^{c, i l l}$ is a $K \times K$ matrix and the components of this matrix are the sensor responses to the illuminations $\mathbf{E}_{c}$ 
and $\mathbf{E}_{i l l}$. As an alternate explanation, sensor response to the illuminants can be understood as the sensor response to a perfect white diffuser under a certain illuminant, and denoted by $\mathbf{w}_{c}$ and $\mathbf{w}_{\text {ill }}$ for the two illuminants. The matrix $\mathbf{D}$ is defined as;

$$
\mathbf{D}^{c, i l l}=\operatorname{diag}\left(\mathbf{w}_{c} / \mathbf{w}_{i l l}\right)
$$

With the use of diagonal transform as in Eq. 7, the problem of color constancy (for 3-channel images) and multispectral constancy (for multispectral data) is reduced to the estimation of $K$ parameters of the diagonal transform. $\mathbf{D}$ is applied on each channel independently and is used in many color constancy algorithms [24, 25].

With a perfect white diffuser, Eq. 5 holds and the input illuminant is transformed into the desired canonical illuminant with a diagonal transform. However, when the surface reflectance is not constant across the wavelength spectrum, then this transformation generates errors. The diagonal transform also works well when the bandwidth of spectral sensitives of filters are within 100 to 150 nanometers $[26,27]$. However, in case of large band filters, the diagonal transform may not be sufficient. Therefore, we investigate the performance of diagonal transform and propose to minimize the error during diagonal transformation through a spectral adaptation transform $\mathbf{A}_{S A T}$. The idea of SAT is to incorporate the sensors response for efficient transformation, along with the diagonal transform. The concept of spectral adaptation transform is analogous to chromatic adaptation transform [28], while here we consider multispectral data, rather than the three-channel color images. 


\subsection{Related state of the art}

To improve the performance of $\mathbf{M}$ for color images, Finlayson et al. [20] proposed sensor sharpening which aims at finding a linear combination of the spectral sensitives of a camera, with respect to which a diagonal transform for illuminant transform works the best. The idea in this technique is to transform the sensor responses so that they appear to be sharper than the original ones. Finlayson et al. [20] proposed three methods for finding the sharpening transform $\mathbf{T}$. The first method is called sensor based sharpening, and finds $\mathbf{T}$ by optimizing the intuitive notion of sharpness for each filter individually. This optimization requires the knowledge of sharpening interval in the wavelength spectrum and the Lagrange multiplier [20]. The second method is called perfect sharpening, in which it is assumed that the surface reflectances can be fitted in a three dimensional linear model while the illuminants can be fitted in a two dimensional linear model. The third method is data based sharpening, in which RGBs are generated from a set of known spectral reflectances, using a specific camera and two illuminations, one as canonical illumination $\mathbf{E}_{c}$ while the other as test illumination $\mathbf{E}_{i l l}$. The sharpening transform $\mathbf{T}$ is added on both sides of Eq. 5 to reduce the error in mapping. It is expressed as;

$$
\mathbf{T F}_{c}=\mathbf{M T F}_{i l l} .
$$

According to Eq. 8, $\mathbf{M}$ can be optimal in the least-square sense if it can be defined by the Moore-Penrose inverse $\left(^{+}\right)$;

$$
\mathbf{M}=\mathbf{T F}_{c}\left[\mathbf{T F}_{i l l}\right]^{+}
$$


Since $\mathbf{T}$ is still unknown in Eq. 9, the unknown terms are shifted to the left hand side;

$$
\mathbf{T}^{-1} \mathbf{M T}=\mathbf{F}_{c}\left[\mathbf{F}_{i l l}\right]^{+}
$$

The sharpening transform $T$ is found through eigenvector decomposition of the expression on left side of Eq. 10. In their work, the expression $[\mathbf{T}]^{-1} \mathbf{M T}$ is diagonalized so that $\mathbf{M}$ is replaced by a diagonal matrix $\mathbf{D}$. Finlayson et al. [20] found similar $\mathbf{T}$ for sensor based sharpening and data based sharpening.

The sharpening transform can sometimes have negative values and there are some investigations to reduce this problem [29]. Data based sharpening was further improved by Barnard et al. [30] by using an average of measured illuminants, and introduced a parameter for prioritizing the positivity. Drew and Finlayson [31] proposed data driven positivity by adding constraints to ensure that all the values in sharpening transform are positive. Chong et al. [32] proposed the measurement tensor technique for finding T. Spherical sampling [33] is also introduced as a technique for spectral sharpening where the discretely sampled points on a sphere are found and related to the original sensors. Sharpening through filter chart calibration was proposed by Abdellatif [34]. An overview of spectral sharpening methods is provided by Corral and Bertalmío [29].

In case of multispectral imaging, the concept of spectral sharpening is not straightforward due to the higher dimensionality of sensors. In [33], spectral sharpening for six multispectral sensors is discussed. Their method is computationally expensive since there is need to generate a sphere of the sensor dimension which is not trivial. For a dimension above 3, the spherical sampling [33] has to be extended to hyper-sphere computation. Due to these complications, we do not use the sensor based sharpening method. We do not use the data based 
sharpening method either, because we are not interested in diagonalizing the result of Eq. 10. Instead, we are using $\mathbf{D}$ as the diagonal matrix containing the illuminant in the sensor domain, as in Eq. 7, and to improve the efficiency of such a transform in the same way as in spectral sharpening. It can be argued that instead of improving the diagonal transform, why not find any other linear transform which can serve the same purpose more efficiently? The reason for preferring the diagonal transform (and its improvement) in our work is the fact that knowledge of scene illuminant is of major importance in computer vision applications. Therefore, either it can be measured for a specific scene or can be estimated, and this information can be used by our proposed method. Another reason is that most of the color constancy algorithms are defined in terms of a diagonal transform, where the elements of diagonal transform are found through illuminant estimation in the sensor domain.

For achieving illuminant invariant representation in multispectral imaging, Abrardo et al. [35] proposed the concept of color constancy for multispectral imaging by linearly transforming the sensor response under an unknown illuminant. The transformation matrix $\mathbf{M}$ is determined through the least-square solution as;

$$
\mathbf{M}=\mathbf{F}_{c} \mathbf{F}_{i l l}^{+}=\mathbf{F}_{c} \mathbf{F}_{i l l}^{t}\left(\mathbf{F}_{i l l} \mathbf{F}_{i l l}^{t}\right)^{-1}
$$

The transformation $\mathbf{M}$ is applied to the acquired multispectral data and then spectral reflectance is estimated. It is interesting to note that the right side of Eq. 11 resembles with the concept of data based sharpening (Eq. 10). This method works well when the canonical representation $\left(\mathbf{F}_{c}\right)$ for the same scene is available. In [35], authors claim that $97 \%$ accuracy in spectral reconstruction is achieved by their method. The problem with this technique is that in the absence of $\mathbf{F}_{c}$, it is not possible to use Eq. 11. In fact, our aim is to transform 
the input multispectral data into its canonical representation, while in Eq. 11, the availability of canonical representation of same scene is assumed, which is not feasible for every image being captured.

\subsection{Proposal for computation of $\mathbf{M}$}

It can be observed that Eq. 10 and 11 are originally formulated from Eq. 5 and the goal is to find the transform $\mathbf{M}$. In spectral sharpening, $\mathbf{M}$ is found by eigenvector decomposition of Eq. 10 and diagonalizing it, while the product of $\mathbf{F}_{c}$ and $\mathbf{F}_{i l l}$ is used directly in Eq. 11. We propose decomposing $\mathbf{M}$ into two elements; $\mathbf{D}^{c, i l l}$ as in Eq. 7 and $\mathbf{A}_{S A T}$. In this proposed method, the diagonal transform performs the transformation of multispectral data taken under unknown illuminant $\mathbf{E}_{i l l}$, into its canonical representation under the illuminant $\mathbf{E}_{c}$. The role of $\mathbf{A}_{S A T}$ is to incorporate the spectral response of imaging sensors and improve the transformation of camera data into its canonical representation. The optimal $\mathbf{A}_{S A T}$ should minimize the error for all reflectances $i$ and illuminants $j$.

$$
\mathbf{F}_{c}^{i}=\mathbf{A}_{S A T} \mathbf{D}^{c, j} \mathbf{F}_{j}^{i}
$$

To find the best $\mathbf{A}_{S A T}$, Eq. 12 is written as the explicit minimization of an error function in Eq. 13;

$$
\min _{\mathbf{A}_{S A T}}=\sum_{j}\left|\mathbf{F}_{c}^{i}-\mathbf{A}_{S A T} \mathbf{D}^{c, j} \mathbf{f}_{j}^{i}\right|
$$

For a given sensor configuration, a generic $\mathbf{A}_{S A T}$ is created by minimizing the error for all the reflectances and illuminations in the training dataset.

The advantage of our proposed technique is that only the knowledge of sensor sensitives of camera is required to compute $\mathbf{A}_{S A T}$. This specific $\mathbf{A}_{S A T}$ for a camera can be used to transform the multispectral data captured under any 
illuminant, into its canonical representation through the diagonal correction for illumination. The only parameter left in the proposed system is the performance of illuminant estimation algorithm, since the elements of diagonal transform are obtained through the illuminant estimation.

The multispectral data being acquired under any illumination is transformed into a canonical representation after estimating the scene illuminant, and then the pre-calibrated $\mathbf{W}_{c}$ is used. The advantage of our proposed idea of multispectral constancy is that it is no more necessary to acquire the multispectral data in a controlled environment.

Once the transform $\mathbf{M}^{c, i l l}=\mathbf{A}_{S A T} \mathbf{D}^{c, i l l}$ is defined, spectral reconstruction can be mathematically represented as;

$$
\hat{\mathbf{R}}=\mathbf{W}_{c} \mathbf{A}_{S A T} \mathbf{D}^{c, i l l} \mathbf{F}_{i l l}
$$

Preliminary results by using the diagonal transform $\mathbf{D}^{c, \text { ill }}$ are provided in [19]. In the current work, we are using $\mathbf{D}^{c, \text { ill }}$ along with $\mathbf{A}_{S A T}$. The experimental protocol for testing the idea of multispectral constancy through SAT is provided in the following section.

\section{Experiments}

To implement and validate the idea of multispectral constancy through SAT, we use measured reflectance data of 1995 surfaces from the SFU reflectance dataset [36]. These surfaces include the 1269 Munsell chips, 24 patches of the X-rite ColorChecker, 170 natural objects [37], 120 Dupont paint chips [37], 350 surfaces from the Krinov dataset and 57 surfaces measured by Barnard et al. [36]. This dataset is used as the training data for calibration of $\mathbf{W}_{c}$ with D65

as the canonical illuminant. The reflectance data is in the wavelength range of 400 to $700 \mathrm{~nm}$ with $10 \mathrm{~nm}$ sampling. 
For testing the proposed idea of multispectral constancy, we use 24 patches from the X-rite ColorChecker and 1296 Munsell chips as the test data and acquire them using the simulated multispectral sensors. Spectral reconstruction is done from the acquired multispectral data as in Eq. 14. To validate the usefulness of proposed idea for natural outdoor images, we use the reflectance data from Foster dataset of hyperspectral images [21]. These hyperspectral images are within wavelength range of $400 \mathrm{~nm}$ to $720 \mathrm{~nm}$, but we use these images within wavelength range of $400 \mathrm{~nm}$ to $700 \mathrm{~nm}$, since the training dataset [36] is within this range. In the following sections, the details of experimental setup are provided.

\subsection{Sensor}

Diagonal transform works well when the sensor sensitives are narrow-band (ideally Dirac delta functions). Following this, it seems as the use of narrow band filters is the optimal choice to be used in imaging systems. The term $\mathbf{F}_{c} \mathbf{F}_{\text {ill }}^{t}$ in Eq. 11 resembles the spectral sharpening, introduced by Finlayson et al. [20]. They proposed a linear combination to transform the original sensor responses into narrower bands. However, from a practical point of view, one reason for not employing such narrow band systems is that the acquisition time, complexity and cost of such system is high as compared to wide band systems [38]. Other reasons are the fact that the narrow band systems are not an optimal choice for illuminant estimation $[16,17]$ and demosaicing (when multispectral filter array is used [39]). Wang et al. [40] studied the influence of increase in number of bands and found that increasing the number of spectral bands cause reduction in performance of spectral reconstruction. Also, the efficiency of illuminant estimation algorithms decreases when the number of spectral filters is increased [17]. In this work, we are limiting the experiments to linear systems and our aim is to investigate the concept of multispectral constancy 
for a generalized multispectral imaging system. We use 5, 8 and 12 spectral bands for testing the proposed framework of spectral constancy. In the results of illuminant estimation in multispectral images by Khan et al. [17], the accuracy of illuminant estimation is reduced when the number of spectral bands is increased beyond 8 . On the other hand, having more than 8 bands still increase the spectral estimation. For this reason, we limit the number of bands within this range for our experiments.

In our experiments, we use a Gaussian model of sensor sensitivities. Such a model has been extensively used in the literature to simulate sensors or to approximate Fabry-Pérot filter transmittance [41]. This configuration is called the

equi-Gaussian [16]. This sensor configuration is tested for illuminant estimation [17] and spectral estimation [19]. The Full Width at Half Maximum (FWHM) of the sensor sensitivities decrease with increase in number of bands and the overlap between adjacent bands remain approximately the same. By increasing the number of bands $(K)$ in this configuration, we are gradually shifting from typical multispectral sensors towards hyperspectral sensors.

For testing the proposed method of SAT for spectral reconstruction, we also use measured sensitivities of a real implementation of spectral filter array (SFA) camera [42]. There are eight filters in this SFA camera. The first six filters are used in our experiments as the available spectral reflectance data for training (SFU dataset) is within the wavelength range of $400 \mathrm{~nm}$ to $700 \mathrm{~nm}$. Figure 1 shows the spectral sensitivities of each filter being used in the experiments.

\section{2. $\mathbf{A}_{S A T}$ computation}

For a given sensor configuration, $\mathbf{A}_{S A T}$ is computed by using the set of 102 illuminants and surface reflectance of 1995 surfaces from the SFU data [36]. Radiance data is generated using an illuminant $j$ (from the set of 102 illuminants) and is acquired as multispectral data. For each illuminant, a diagonal 


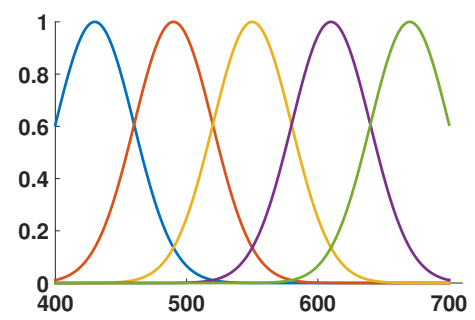

(a) Equi-Gaussian filters $(K=5)$

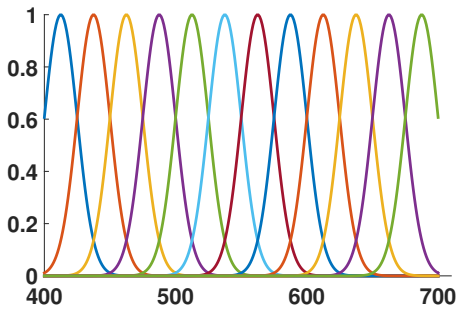

(c) Equi-Gaussian filters $(\mathrm{K}=12)$

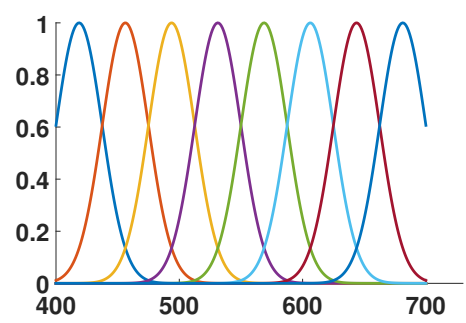

(b) Equi-Gaussian filters $(\mathrm{K}=8)$

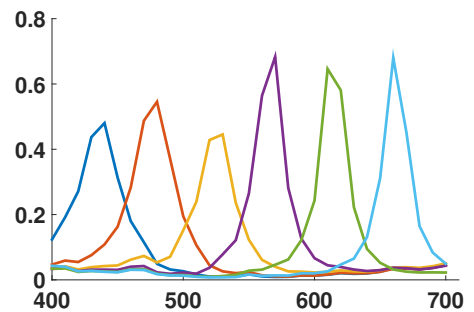

(d) 6 sensors from the SFA

Figure 1: Spectral sensitivities of the filters being used

transform is created by using Eq. 7 and the multispectral data under that illuminant is transformed by using Eq. 5. The desired output after applying $\mathbf{M}^{c, \text { ill }}$ is the multispectral data under the canonical illuminant (D65). As discussed in Section 2.3, we propose to decompose $\mathbf{M}^{c, i l l}$ into diagonal transform $\mathbf{D}^{c, i l l}$, and $\mathbf{A}_{S A T}$. This $\mathbf{A}_{S A T}$ is unique for a given sensor configuration and is computed by using Eq. 13. Once $\mathbf{A}_{S A T}$ is computed, Eq. 12 is used to transform $\mathbf{F}_{\text {ill }}$ into $\mathbf{F}_{c}$. $\mathbf{A}_{S A T}$ for each sensor configuration being tested in this work is provided in the supplementary data file (Supplementary_Data.xlsx).

\subsection{Illuminant estimation}

For testing and validating the proposed concept of spectral adaptation transform, we keep $\mathbf{D}^{\text {cill }}$ fixed for the spectral reflectance data. For obtaining the elements of $\mathbf{D}^{c, \text { ill }}$, we use the test and canonical illuminants in the sensor domain. Initially we want to validate the efficiency of $\mathbf{A}_{S A T}$ while testing the reflectance dataset, therefore we assume that the illuminant is estimated in the 
sensor domain without error.

In practical cases, the information about scene illumination may not be available all the time. This is where the illumination estimation algorithms come into play. By using an illuminant estimation algorithm, the elements of diagonal transform are obtained and then the pre-calculated SAT is used.

For testing the proposed concept for natural scenes, we perform illuminant estimation in the multispectral data of natural scenes and use the Max-Spectral Algorithm, which is the extension of Max-RGB algorithm [43]. The extension of this algorithm from color to spectral is proposed and analyzed in detail in $[16,17]$. The estimated values of illuminant, which are defined in the sensor domain, are used in the diagonal transform $\mathbf{D}^{c, i l l}$. For each image, $\mathbf{D}^{c, i l l}$ is estimated individually and this estimation may consist of error as well. We report the error in illuminant estimation in form of angular error $(\Delta A)$.

\subsection{Spectral reflectance reconstruction}

As explained in Section 2, a calibration matrix $\mathbf{W}$ is required for the spectral reconstruction from camera data. It is obtained by using measured reflectance spectra $\mathbf{R}_{t}$ and the camera sensor sensitivities $(\mathbf{M})$. For reducing the error be-

tween original spectra $\mathbf{R}$ and the estimated spectra $\hat{\mathbf{R}}$, a covariance matrix of a set of measured reflectance samples can be used. Those measured reflectance samples provide the a-priori statistical information about the surfaces in a scene [44]. If the a-priori information is well chosen, error in the spectral reconstruction can be minimal.

There are several methods being proposed for the spectral reconstruction in literature [45]. We use a linear method for clarity, namely the Wiener estimation [46] because of its robustness to noise. It is defined as

$$
\mathbf{W}=\mathbf{R}_{t} \mathbf{R}_{t}^{T}(\mathbf{C E})^{T}\left((\mathbf{C E}) \mathbf{R}_{t} \mathbf{R}_{t}^{T}(\mathbf{C E})^{T}+\mathbf{G}\right)^{-1}
$$


Here, $\mathbf{R}_{t} \mathbf{R}_{t}^{T}$ and $\mathbf{G}$ are the autocorrelation matrices of the training spectra and additive noise, respectively. $\mathbf{G}$ is in the form of a diagonal matrix consisting of the variance of noise $\sigma^{2}$. Training for obtaining the matrix $\mathbf{W}_{c}$ is performed with CIE illuminant D65 as the canonical illuminant $\mathbf{E}_{c}$. The obtained calibration matrix is used for spectral reconstruction in Eq. 14.

\subsection{Evaluation}

For testing the proposed idea of multispectral constancy, radiance data is simulated from the measured test spectra with CIE illuminants A, D50, D55, D75, F5, F7 and F12. We also use the LED (Philips SlimStyle: 2700K) as an illuminant in the experiments.

The proposed idea is also tested on hyperspectral images of scenes consisting of vegetation and urban areas. We create radiance data from these images using illuminants A, D50, D55 and D75. The same procedure of reflectance reconstruction for each pixel of simulated multispectral image is performed and evaluated.

To measure the performance of the spectral reconstruction, we compare the reconstruction $\hat{\mathbf{r}}$ for each patch of the reconstructed reflectance with the corresponding measured reflectance $\mathbf{r}$, through root mean square error (RMSE) as

$$
\mathrm{RMSE}=\sqrt{\frac{1}{N} \sum_{j=1}^{N}\left(r_{j}-\hat{r}_{j}\right)^{2}}
$$

We also use goodness of fit coefficient (GFC) [47] for evaluation of spectral reconstruction results. For each reconstructed reflectance $\hat{\mathbf{r}}$, GFC is calculated 


$$
\mathrm{GFC}=\frac{\mathbf{r}^{T} \hat{\mathbf{r}}}{\sqrt{\left(\mathbf{r}^{T} \mathbf{r}\right)\left(\hat{\mathbf{r}}^{T} \hat{\mathbf{r}}\right)}}
$$

In case of the multispectral images from Foster dataset, RMSE is calculated for each pixel of the estimated reflectance, with the original reflectance in the hyperspectral image. We also compute the colorimetric error by a linear mapping of the reflectance data into its corresponding CIEXYZ. The CIEXYZ is converted into CIELab by taking white point of D65. Error between CIELab from the original reflectance and reconstructed reflectance is calculated in terms of CIEDE2000 [48]. For each evaluation metric, we include three methods. First method is by doing nothing to the input multispectral data and using Eq. 15 for spectral reconstruction. We call this method as do nothing. In second method, simple diagonal transform is applied to the input data before using it for spectral reconstruction, while in the third experiment, the input data is first transformed by using the proposed SAT. Results are discussed in Section 4.

\section{Results}

The results of spectral reconstruction of Munsell reflectance data, by using eight different illuminations and four different sensor configurations, are shown in form of graphs in Fig. 2, consisting of mean RMSE, mean GFC and mean CIEDE2000. For each test illuminant, we compare the spectral reconstruction results after applying diagonal transform to the input multispectral data, SAT and do nothing. The RMSE results show that for illuminant $\mathrm{A}$, the diagonal transform reduces the error as comparing to do nothing, but for illuminants D50, D55 and D75, applying only a simple diagonal transform significantly increases the error in spectral reconstruction as compared to when the SAT is applied. There is slight increase in RMSE with the use of diagonal transform 
for illuminants F5, F7 and F12, while there is no change in error when LED is used as illuminant source. These results show that in terms of RMSE, applying diagonal transform to input multispectral data increases the error in comparison to do nothing. This trend is consistent among the 5,8 and 12 channel cameras being used. Results obtained from simulated 6 channel SFA camera also show similar results. When our proposed SAT is used along with diagonal transform on the input multispectral data, then RMSE is reduced significantly. By increasing the number of channels, there is further reduction in RMSE for all illuminants except F12, where RMSE is minimum when 8 channels are used. Overall, the significant reduction in RMSE shows the efficiency of our proposed SAT for spectral reconstruction.

When the spectral reconstruction is evaluated in terms of GFC, diagonal transform and SAT perform closely. Do nothing performs lower for illuminants A, F5, F12 and LED while the performance difference is less significant for other test images. There is no change in performance by increasing the number of channels, except for illuminant F12, where the performance of do nothing goes down by increasing the number of channels. The increase in error for do nothing is because the bands become more sensitive to illumination changes when they get narrower.

Colorimetric evaluation of the spectral reconstruction reveals the interesting fact that there is no difference in do nothing and applying the diagonal transform. Error in terms of CIEDE2000 for both diagonal transform and do nothing, is increased slightly when the number of channels are increased from 5 to 12 . However, applying SAT significantly reduces the colorimetric error, as can be seen in Fig. 2. The performance of SAT behaves opposite to other two methods and error is slightly reduced with increase in number of channels.

For testing the proposed idea of multispectral constancy on images contain- 


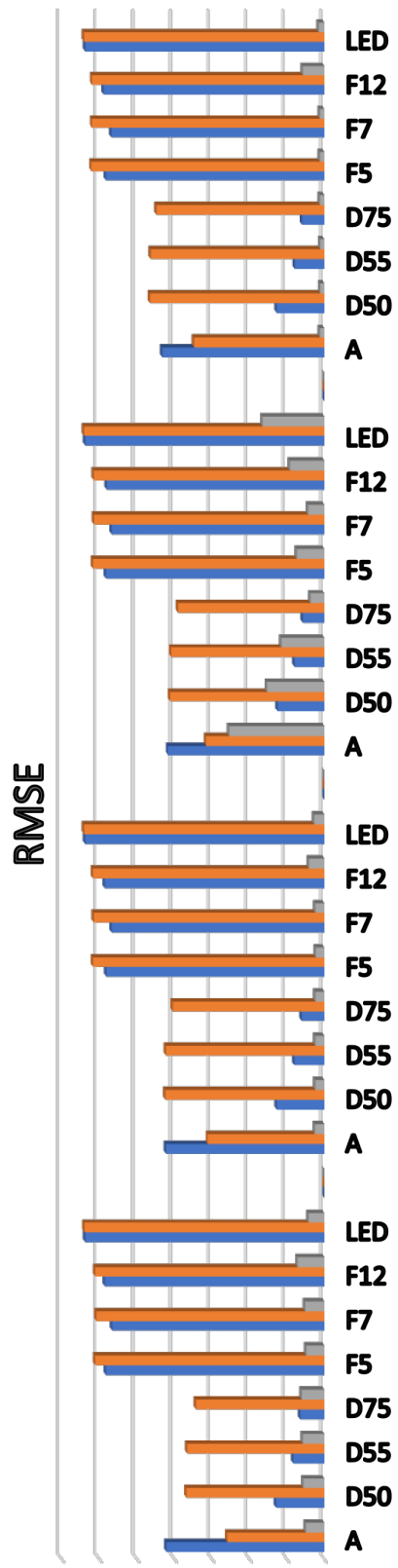

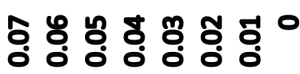
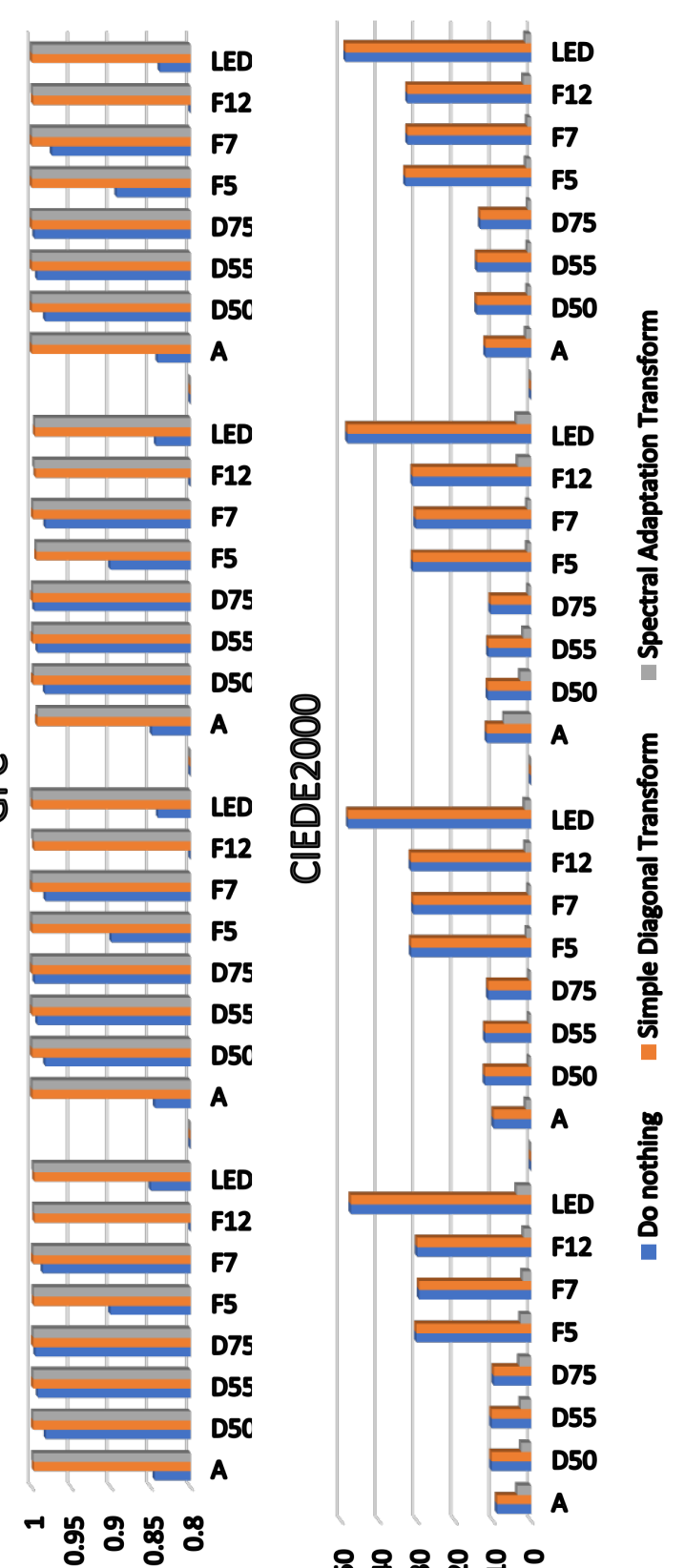

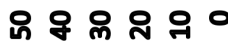

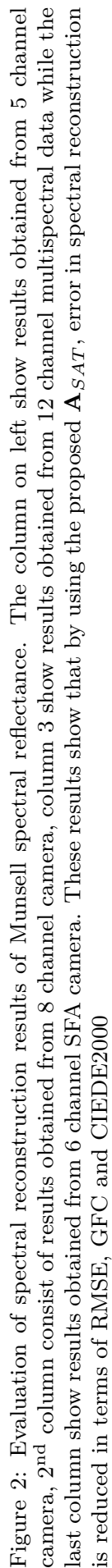


ing real scenes, we use the Foster hyperspectral reflectance data [21]. We use four different sensor configurations as simulation of multispectral camera for acquiring the data. Sensor configuration for these cameras is shown in Fig. 1. Before acquiring the multispectral data, each hyperspectral reflectance image is converted into radiance image by applying an illuminant. We use illuminants A, D50, D55 and D75.

Illuminant estimation in each multispectral image is performed by using the Max-spectral algorithm [17]. Diagonal transform is applied to each multispectral image with the estimated illuminant. That estimated illuminant contain error which is evaluated in terms of angular error $(\Delta A)$. Fig. 3 show the performance of spectral reconstruction in terms of mean RMSE, mean GFC and mean CIEDE2000 of the eight images being tested. Detailed results along with $\Delta A$ are provided in the supplementary data file.

With the Foster dataset, the error in spectral reconstruction is larger than the results of Munsell dataset. The main reason is the error in illuminant estimation. As the elements of diagonal transform are an estimation of the illuminant, therefore the error in illuminant estimation is intensified in the spectral reconstruction as well. Fig. 3 show RMSE results for 5, 8, 12, and 6 (SFA) channels, respectively. Do nothing and simple diagonal transform produces almost similar results except for multispectral data acquired with 8 channels. In that case, simple diagonal transform performs better as compared to do nothing. RMSE is reduced significantly when SAT is used, which shows that our proposed method performs effectively in reducing RMSE.

In terms of GFC, do nothing performs better than simple diagonal transform, except for illuminant A, where the do nothing performs significantly lower. Same trend can be seen across all four sensor configurations being tested. SAT is able to perform slightly better than diagonal transform and do nothing. Evaluation 
in terms of CIEDE2000 shows that do nothing and simple diagonal transform performs same except for 5 channels, where error is increased when diagonal transform is used. By using the proposed SAT, there is decrease in CIEDE2000 which shows that the proposed idea is valid with images containing real scenes. Although the colorimetric error is still large, but it should be kept in mind that inaccuracy in illuminant estimation also play its role in the overall error in spectral reconstruction. The average RMSE with 5 and 12 channels are almost equal and the same result in obtained with SFA, while RMSE is comparatively larger when 8 channels are used. In terms of colorimetry, the error is reduced gradually by increasing the number of channels. When simple diagonal transform is used then CIEDE2000 remains almost equal for 8 and 12 channels while the error is reduced for all sensor configurations when SAT is used. Therefore, by increasing the number of filters, there is slight improvement in the spectral reconstruction results. However, the performance may become more sensitive to the imaging noise. Those results are based on simulations and are still to be validated for the experimentally captured multispectral data. Detailed results from the experiments, including the angular error in illuminant estimation for each image, mean and maximum errors of RMSE, GFC and CIEDE2000, are provided in the supplementary data file.

In most of the illuminant estimation algorithms proposed in literature, the efficiency of algorithm is evaluated in the terms of angular error $\Delta A$ and a diagonal transform is applied to the input images. In most of the cases, the transformed images appear to be taken under a canonical (usually white) illuminant and it is assumed that the effect of scene illuminant is removed through the diagonal transform. Results from our experiments show that a simple diagonal transform is not sufficient. For illustration, we show an example of radiance image from Foster dataset and the corresponding transformations (Fig. 8. In 


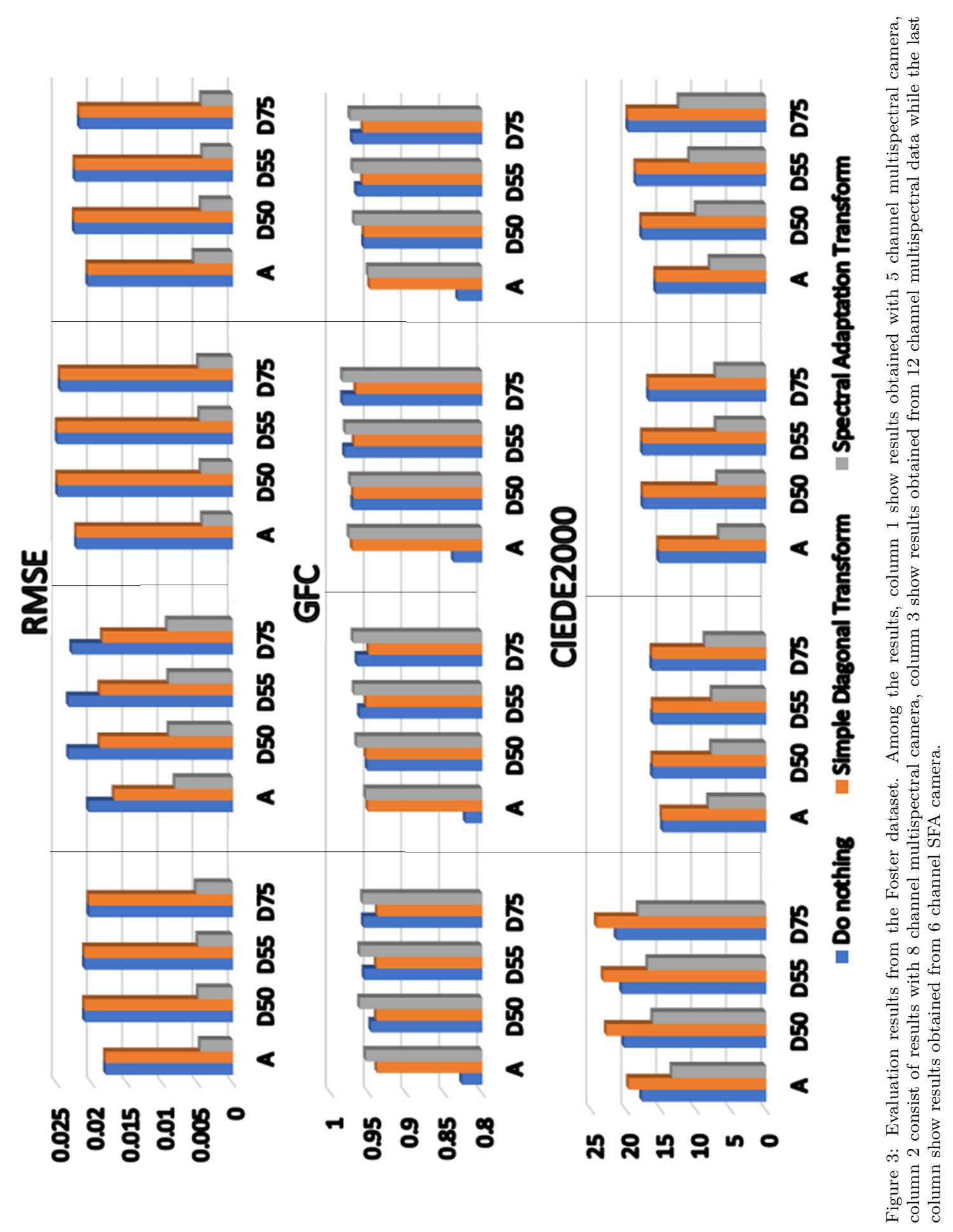


this example, the color rendering of radiance spectral image with test illumination and reconstructed spectral images (after applying diagonal transform and SAT), are shown. D55 is used for creating the radiance scene and multispectral image is acquired with 12 equi-Gaussian channels Illuminant estimation gives $\Delta A$ of 0.1013 . As can be seen in Fig. 8, the effect due to D55 is removed after the transformations and the output images appear to be almost the same visually but the RMSE, GFC and CIEDE2000 evaluation shows that there is difference among these 2 images in terms of spectra and colorimetry. This suggests that the effect of such transformations has to be verified in computer vision applications.

To visualize the reconstructed spectra, eight examples are provided in Fig. 4, 5, 6 \& 7. In each Fig. two sample spectra for one image of Foster dataset are shown. These samples are selected on the basis of best and worst GFC values. Each Fig. provides the comparison between the measured spectral reflectance and the spectral reconstruction results with do nothing, simple diagonal transform and SAT. For each figure, the measured values of $\Delta A$, GFC and RMSE are provided in the captions.

The reconstruction results using simple diagonal transform and SAT match closely, while the do nothing results are significantly lower, as they are not close to the measured spectra in terms of shape and scale. This observation shows that with illuminant estimation and then applying the transformation into canonical representation has the clear advantage. Upon a careful comparison, it can be seen that by applying SAT, the scale of reconstructed spectra is brought closer to the original spectra. This explains the reason that why our proposed SAT is able to reduce the error in spectral reconstruction. The visualization of spectra shows that with the use of SAT, there is no significant difference in the shape of reconstructed spectra. The main difference is in scale of intensity, which explains 

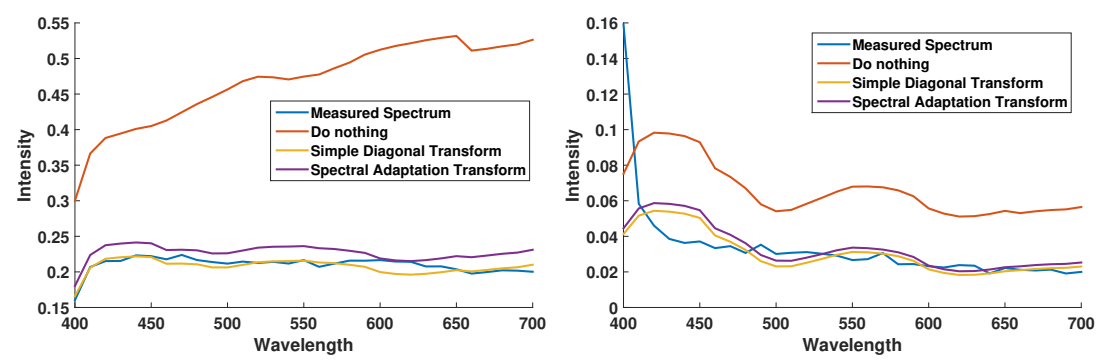

(a) GFC with simple diagonal transform: (b) GFC with simple diagonal transform: 0.9994, GFC with SAT: 0.9994. 0.8463. GFC with SAT: 0.8466 RMSE with simple diagonal transform: RMSE with simple diagonal transform: 0.0489, RMSE with SAT: $0.0033 \quad 0.0124$ RMSE with SAT 0.0039

Figure 4: Spectral reconstruction of 2 sample spectra from Foster dataset (image 7). Illuminant D55 is used to create radiance data, simulated multispectral camera with 5 equi-Gaussian filters is used and illuminant estimation error $\Delta A$ is 0.0162

that why GFC remains same while RMSE and CIEDE2000 metrics provide different results. One of the drawbacks of using linear method for spectral reconstruction is its limitation in reconstruction of spectra containing spikes. Linear spectral reconstruction assumes smooth spectra and provides a smoothed approximation to the input spiky spectra. This effect can be seen in Fig. 4b, $5 \mathrm{~b}, 6 \mathrm{~b} \& 7 \mathrm{~b}$, where the linear reconstruction is unable to address the spikes in the measured spectra.

These observations lead to the question that what is the purpose of color and multispectral constancy. If it has to be applied for visual correction only, then a simple diagonal transform may be efficient, as seen in Fig. 8. If the purpose is to reconstruct the spectral reflectance, then a simple diagonal transform is not helpful while GFC metric indicates that there is slight improvement when simple diagonal transform is used. Evaluation in terms of CIEDE2000 indicates that the results with do nothing and diagonal transform are the same. However, all the evaluation metrics show that the proposed SAT is able to reduce the error significantly. Having said this, the next goal is to investigate that what is the role of improving the currently used evaluation metrics in computer vision 

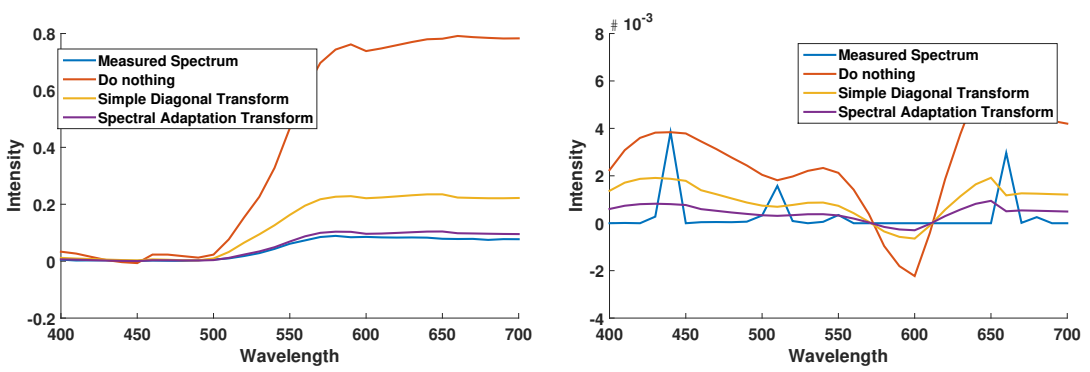

(a) GFC with simple diagonal transform: (b) GFC with simple diagonal transform: 0.9985 , GFC with SAT: 0.9990. 0.3848 , GFC with SAT: 0.3956 .

RMSE with simple diagonal transform: RMSE with simple diagonal transform: 0.0562, RMSE with SAT: 0.0023 0.0013, RMSE with SAT: 0.0001

Figure 5: Spectral reconstruction of 2 sample spectra from Foster dataset (image 4). Illuminant D50 is used to create radiance data, simulated multispectral camera with 8 equi-Gaussian filters is used and illuminant estimation error $\Delta A$ is 0.0373
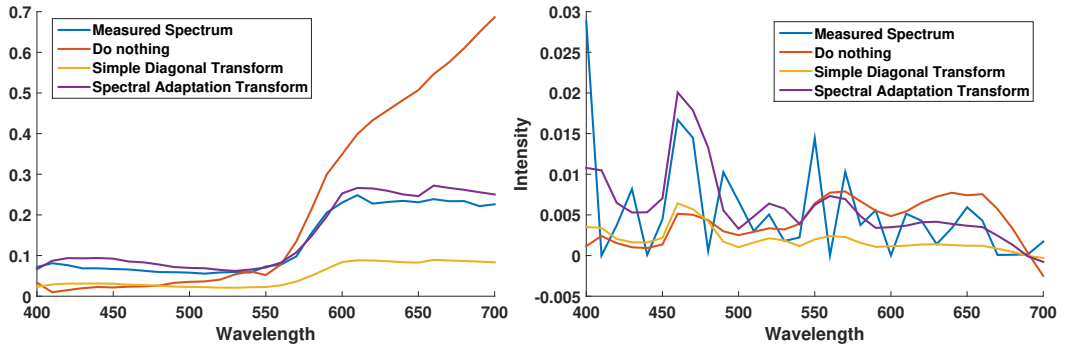

(a) GFC with simple diagonal transform: (b) GFC with simple diagonal transform: 0.9977, GFC with SAT: 0.9981. 0.7652, GFC with SAT: 0.7666 .

RMSE with simple diagonal transform: RMSE with simple diagonal transform: 0.0202, RMSE with SAT: $0.0036 \quad 0.0036$, RMSE with SAT: 0.0013

Figure 6: Spectral reconstruction of 2 sample spectra from Foster dataset (image 6). Illuminant $\mathrm{A}$ is used to create radiance data, simulated multispectral camera with 12 equi-Gaussian filters is used and illuminant estimation error $\Delta A$ is 0.0545 

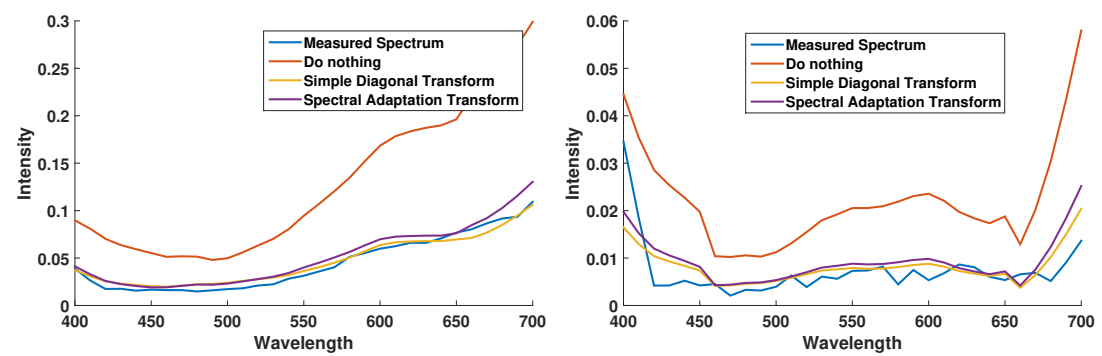

(a) GFC with simple diagonal transform: (b) GFC with simple diagonal transform: 0.9946, GFC with SAT: 0.9971. 0.8832 , GFC with SAT: 0.8840 .

RMSE with simple diagonal transform: RMSE with simple diagonal transform: 0.0183, RMSE with SAT: $0.0015 \quad 0.0036$, RMSE with SAT: 0.0008

Figure 7: Spectral reconstruction of 2 sample spectra from Foster dataset (image 8). Illuminant D75 is used to create radiance data, simulated multispectral camera with 6 SFA channels is used and illuminant estimation error $\Delta A$ is 0.0884
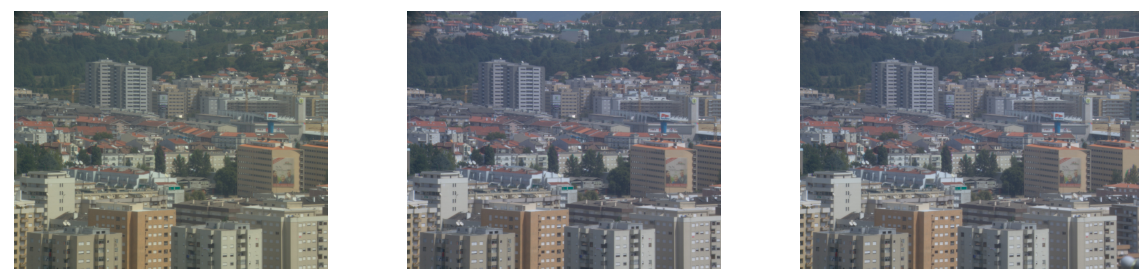

Figure 8: Color rendering of multispectral image acquired with 12 channels. Images from left to right: Rendering under illuminant D55, Diagonal transform, SAT. $\Delta A$ is 0.1013

applications, such as object detection and material identification.

\section{Conclusion and perspectives}

In this work, we propose the concept of multispectral constancy, which defines the illuminant invariant representation of multispectral images. Multispectral constancy is achieved through a spectral adaptation transform (SAT), which transforms the data representation from an unknown illuminant, to a canonical one. This SAT is obtained by training the system. Advantage of the proposed technique is that the only information required is the sensor sensitivities of imaging system. 
With the idea of multispectral constancy being developed, what needs further attention is the establishment of efficient illuminant estimation methods for multispectral imaging. The proposed framework for spectral reconstruction is sensitive to accuracy in illuminant estimation. This dependency is seen when spectral reconstruction is performed on the images from Foster dataset. The performance of SAT is affected with error in illuminant estimation and this dependency may be addressed through development of efficient illuminant estimation algorithms. Once such an efficient illuminant estimation algorithm in the sensor domain is available, our proposed SAT is able to show efficient results as demonstrated in the simulations.

The colorimetric difference also needs to be investigated so that an acceptable level of accuracy for color based systems can be achieved. Finally, it has yet to be investigated that what level of accuracy is required in the spectral reconstruction so that the estimation can be used for spectral information based applications. Such an application can be object detection and identification on the basis of its spectral properties. Hence. our proposed idea of multispectral constancy is opening research questions which needs to be investigated, in order to enable the generic use of multispectral imaging for real-world applications. Improvement in results can be done by carefully selecting the training data which can represent the objects in the scene. In this way, the SAT and spectral reconstruction system can be calibrated for a specific scenario. We did not work on improvement in selection of training data in this work as our main aim is to study the effectiveness of proposed idea of multispectral constancy and SAT under general conditions.

Simulation results show that for unimodal sensitivities and linear sensor, the proposed SAT is an adequate transform and permits to achieve efficient reflectance reconstruction. However, when the SAT is evaluated based on an 
estimate of illumination, error in illuminant estimates makes the performance to decrease. It is still to be investigated that what accuracy is required for illuminant estimation to make this concept beneficial. Further work shall investigate these directions and define the limits of using this approach.

\section{References}

[1] D. H. Foster, "Color constancy," Vision Research, vol. 51, no. 7, pp. 674700, 2011. Vision Research 50th Anniversary Issue: Part 1.

[2] A. Gijsenij, T. Gevers, and J. Van De Weijer, "Computational color constancy: Survey and experiments," IEEE Transactions on Image Processing, vol. 20, pp. 2475-2489, sep 2011.

[3] K. Barnard, V. Cardei, and B. Funt, "A comparison of computational color constancy algorithms - Part I: Methodology and experiments with synthesized data," IEEE Transactions on Image Processing, vol. 11, pp. 972-984, sep 2002.

[4] S. D. Hordley, "Scene illuminant estimation: Past, present, and future," Color Research $\mathscr{E}$ Application, vol. 31, no. 4, pp. 303-314, 2006.

[5] F. H. Imai and R. S. Berns, "Spectral estimation using trichromatic digital cameras," in Proceedings of the International Symposium on Multispectral Imaging and Color Reproduction for Digital Archives, pp. 1-8, Chiba University Chiba, Japan, 1999.

[6] J. Y. Hardeberg, Acquisition and Reproduction of Color Images: Colorimetric and Multispectral Approaches. Universal Publishers, 2001.

[7] D. Connah, S. Westland, and M. G. A. Thomson, "Recovering spectral information using digital camera systems," Coloration Technology, vol. 117, pp. 309-312, nov 2001. 
[8] E. M. Valero, J. L. Nieves, S. M. C. Nascimento, K. Amano, and D. H. Foster, "Recovering spectral data from natural scenes with an RGB digital camera and colored filters," Color Research 83 Application, vol. 32, no. 5, pp. $352-360,2007$.

[9] J. Y. Hardeberg and R. Shrestha, "Multispectral colour imaging: Time to move out of the lab?," in Mid-term meeting of the International Colour Association (AIC), (Tokyo, Japan), pp. 28-32, May 2015.

[10] L. T. Maloney, "Evaluation of linear models of surface spectral reflectance with small numbers of parameters," J. Opt. Soc. Am. A, vol. 3, pp. 16731683 , oct 1986 .

[11] L. T. Maloney and B. A. Wandell, "Color constancy: a method for recovering surface spectral reflectance," J. Opt. Soc. Am. A, vol. 3, pp. 29-33, Jan 1986.

[12] R. Hall, Illumination and color in computer generated imagery. Monographs in Visual Communication, New York, NY: Springer New York, 1989.

[13] J. P. S. Parkkinen, J. Hallikainen, and T. Jaaskelainen, "Characteristic spectra of Munsell colors," J. Opt. Soc. Am. A, vol. 6, pp. 318-322, feb 1989.

[14] T. Jaaskelainen, J. Parkkinen, and S. Toyooka, "Vector-subspace model for color representation," J. Opt. Soc. Am. A, vol. 7, pp. 725-730, apr 1990.

[15] R. Shrestha and J. Y. Hardeberg, "Spectrogenic imaging: a novel approach to multispectral imaging in an uncontrolled environment.," Optics Express, vol. 22, pp. 9123-33, apr 2014.

[16] J.-B. Thomas, "Illuminant estimation from uncalibrated multispectral im- 
ages," in Colour and Visual Computing Symposium (CVCS), (Gjøvik, Norway), pp. 1-6, Aug 2015.

[17] H. A. Khan, J. B. Thomas, J. Y. Hardeberg, and O. Laligant, "Illuminant estimation in multispectral imaging," J. Opt. Soc. Am. A, vol. 34, pp. 10851098, Jul 2017.

[18] G. D. Finlayson, M. S. Drew, and B. V. Funt, "Color constancy: generalized diagonal transforms suffice," J. Opt. Soc. Am. A, vol. 11, pp. 3011-3019, Nov 1994.

[19] H. A. Khan, J. B. Thomas, and J. Y. Hardeberg, "Multispectral constancy based on spectral adaptation transform," in 20th Scandinavian Conference on Image Analysis, SCIA, Tromsø, Norway, June 12-14, 2017, Proceedings, Part II (P. Sharma and F. M. Bianchi, eds.), pp. 459-470, Springer International Publishing, 2017.

[20] G. D. Finlayson, M. S. Drew, and B. V. Funt, "Spectral sharpening: sensor transformations for improved color constancy," J. Opt. Soc. Am. A, vol. 11, pp. 1553-1563, may 1994.

[21] D. H. Foster, K. Amano, S. M. C. Nascimento, and M. J. Foster, "Frequency of metamerism in natural scenes," J. Opt. Soc. Am. A, vol. 23, p. 2359, oct 2006.

[22] Y. Zhao and R. S. Berns, "Image-based spectral reflectance reconstruction using the matrix R method," Color Research 83 Application, vol. 32, pp. 343-351, oct 2007.

[23] N. Eslahi, S. H. Amirshahi, and F. Agahian, "Recovery of spectral data using weighted canonical correlation regression," Optical Review, vol. 16, pp. 296-303, may 2009. 
[24] G. D. Finlayson and E. Trezzi, "Shades of gray and colour constancy," Proceedings of the Twelfth Color Imaging Conference, pp. 37-41, 2004.

[25] J. van de Weijer, T. Gevers, and A. Gijsenij, "Edge-based color constancy," IEEE Transactions on Image Processing, vol. 16, pp. 2207-2214, sep 2007.

[26] J. A. Worthey and M. H. Brill, "Heuristic analysis of Von Kries color constancy," J. Opt. Soc. Am. A, vol. 3, pp. 1708-1712, Oct 1986.

[27] D. A. Forsyth, "A novel algorithm for color constancy," International Journal of Computer Vision, vol. 5, pp. 5-35, aug 1990.

[28] R. W. G. Hunt, C. Li, and M. R. Luo, "Chromatic adaptation transforms," Color Research \&3 Application, vol. 30, no. 1, pp. 69-71, 2005.

[29] J. Vazquez-Corral and M. Bertalmío, "Spectral sharpening of color sensors: Diagonal color constancy and beyond," Sensors, vol. 14, pp. 3965-3985, feb 2014 .

[30] K. Barnard, F. Ciurea, and B. Funt, "Sensor sharpening for computational color constancy.," J. Opt. Soc. Am. A, vol. 18, pp. 2728-43, nov 2001.

[31] M. Drew and G. Finlayson, "Spectral sharpening with positivity," J. Opt. Soc. Am. A, vol. 17, no. 8, pp. 1361-70, 2000.

[32] H. Y. Chong, S. J. Gortler, and T. Zickler, "The von Kries hypothesis and a basis for color constancy," in Proceedings of the IEEE International Conference on Computer Vision, pp. 1-8, IEEE, 2007.

[33] G. D. Finlayson, J. Vazquez-Corral, S. Süsstrunk, and M. Vanrell, "Spectral sharpening by spherical sampling," J. Opt. Soc. Am. A, vol. 29, no. 7, p. 1199, 2012. 
[34] M. Abdellatif, "Physics-based spectral sharpening through filter-chart calibration," Color Research $\& 3$ Application, vol. 40, pp. 564-576, dec 2015.

[35] A. Abrardo, L. Alparone, I. Cappellini, and A. Prosperi, "Color constancy from multispectral images," International Conference on Image Processing, vol. 3, pp. 570-574, 1999.

[36] K. Barnard, L. Martin, B. Funt, and A. Coath, "A data set for color research," Color Research E3 Application, vol. 27, no. 3, pp. 147-151, 2002.

[37] M. J. Vrhel, R. Gershon, and L. S. Iwan, "Measurement and analysis of object reflectance spectra," Color Research \& Application, vol. 19, no. 1, pp. 4-9, 1994.

[38] R. Shrestha and J. Y. Hardeberg, "Evaluation and comparison of multispectral imaging systems," in Color and Imaging Conference, pp. 107-112, 2014.

[39] P. J. Lapray, X. Wang, J. B. Thomas, and P. Gouton, "Multispectral filter arrays: Recent advances and practical implementation," Sensors, vol. 14, pp. 21626-21659, nov 2014.

[40] X. Wang, J.-B. Thomas, J. Y. Hardeberg, and P. Gouton, "Multispectral imaging: narrow or wide band filters?," Journal of the International Colour Association, vol. 12, pp. 44-51, 2014.

[41] P. J. Lapray, J. B. Thomas, P. Gouton, and Y. Ruichek, "Energy balance in spectral filter array camera design," Journal of the European Optical Society, vol. 13, p. 1, dec 2017.

[42] J. B. Thomas, P. J. Lapray, P. Gouton, and C. Clerc, "Spectral characterization of a prototype SFA camera for joint visible and NIR acquisition," Sensors, vol. 16, p. 993, jun 2016. 
[43] E. H. Land and J. J. McCann, "Lightness and Retinex Theory," Journal of the Optical Society of America, vol. 61, pp. 1-11, jan 1971.

[44] J. Conde, H. Haneishi, M. Yamaguchi, N. Ohyama, and J. Baez, "Spectral reflectance estimation of ancient Mexican codices, multispectral images approach," Revista Mexicana de Fisica, vol. 50, no. 5, pp. 484-489, 2004.

[45] D. Connah, J. Y. Hardeberg, and S. Westland, "Comparison of linear spectral reconstruction methods for multispectral imaging," in International Conference on Image Processing, ICIP, vol. 3, pp. 1497-1500, 2004.

[46] H.-L. Shen, P.-Q. Cai, S.-J. Shao, and J. H. Xin, "Reflectance reconstruction for multispectral imaging by adaptive Wiener estimation," Optics Express, vol. 15, no. 23, pp. 15545-15554, 2007.

[47] J. Hernández-Andrés, J. Romero, and R. L. Lee, "Colorimetric and spectroradiometric characteristics of narrow-field-of-view clear skylight in Granada, Spain,” J. Opt. Soc. Am. A, vol. 18, pp. 412-420, Feb 2001.

[48] CIE, Improvement to industrial colour-difference evaluation. CIE technical report 142-2001, Vienna: Central Bureau of the CIE, 2001. 